\title{
PHÂN LẠP VÀ XÁC ĐỊNH CẤU TRÚC CỦA MộT SỐ DẪN XUẤT NAPHTHOPYRONE VÀ ANTHRAQUINONE TỬ CỦ SÂM ĐẠI HÀNH (Eleutherine bulbosa)
}

\author{
Nguyễn Thị Hồng Vân ${ }^{1, ~ *, ~ L u ̛ u ~ T u a ̂ ́ n ~ A n h ~}{ }^{1}$, Lê Minh Hà1 ${ }^{1}$ Ngô Thị Phương ${ }^{1}$, \\ Trịnh Anh Viên², Phạm Quốc Long ${ }^{1}$ \\ ${ }^{1}$ Viện Hóa học các Hợp chất thiên nhiên, Viện HLKHCNVN, \\ 18 Hoàng Quốc Việt, Cầu Giấy, Hà Nội. \\ ${ }^{2}$ Truoòng Đại học Công nghiệp thành phố Hồ Chỉ Minh - co sở Thanh Hóa \\ "Email:van762004@yahoo.com
}

Đến Toà soạn: 01/7/2012; Chấp nhận đăng: 31/8/2013

\section{TÓM TẮT}

Hai dẫn xuất naphthopyrone là eleutherinol (1) và eleutherinoside $\mathrm{A}$ (2) đã được phân lập từ cặn chiết etyl axetat của củ sâm đại hành (Eleutherine bulbosa) cùng với một dẫn xuất anthraquinone là 1,3,6-trihydroxy-8-methyl-anthraquinone (3). Cấu trúc của cả 3 hợp chất này đã được xác định dựa vào các phương pháp phổ cộng hưởng từ hạt nhân 1 chiều và 2 chiều.

Tù khoá: sâm đại hành, naphthopyrone, eleutherinol, eleutherinoside $\mathrm{A}$, anthraquinone, 1,3,6trihydroxy-8-methyl-anthraquinone, Eleutherine bulbosa.

\section{MỞ ĐẦU}

Cây Sâm đại hành (Eleutherine bulbosa (Mill.) Urban) thuộc họ Lay dơn (Iridaceae), là loại cây thảo cao $20-30 \mathrm{~cm}$, thân hành, có củ màu đỏ. Ở Việt Nam, cây mọc hoang ở nhiều nơi, dân gian thường sử dụng củ sấy khô làm thuốc uống chữa các chứng bệnh thiếu máu, vàng da, hoa mắt, nhức đầu, tiêu viêm, ho lao, ho gà, thương tích lưu huyết, viêm họng cấp và mạn, tê bại do thiếu dinh dưỡng, đinh nhọt, viêm da, lở ngứa, chốc đầu trẻ em, tổ đỉa, vẩy nến. Loài này cũng phổ biến ở vùng Nam Mỹ và các nước khu vực Đông Nam Á và thường được dùng trong y học dân gian để chữa trị các chứng bệnh về tim và phục hồi vết thương $[1,2]$. Các nghiên cứu trước đây cho thấy củ sâm đại hành có chứa các hợp chất naphthoquinon cùng một số dẫn xuất của anthraquinon và naphthalen $[3,4]$. Các hợp chất này thể hiện những hoạt tính sinh học đáng chú ý như kháng khuẩn, kháng nấm, kháng viêm giảm đau, gây độc tế bào... [5]. Trong chương trình nghiên cứu về thành phần hóa học củ sâm đại hành, chúng tôi đã phân lập được một số hợp chất naphthopyran từ củ sâm đại hành [6]. Trong bài báo này, chúng tôi tiếp tục trình bày việc phân lập và xác định cấu trúc của hai dẫn xuất của naphthopyrone là eleutherinol (1) và eleutherinoside $\mathrm{A}$ (2) cùng một dẫn xuất của anthraquinone là 1,3,6-trihydroxy-8-methylanthraquinone (3) từ củ sâm đại hành. 


\section{NGUYÊN LIỆU VÀ PHƯƠNG PHÁP NGHIÊN CÚU}

\subsection{Nguyên liệu}

Củ sâm đại hành (Eleutherin bulbosa) được thu hái tại Chí Linh, Hải Dương vào tháng 2 năm 2012. Mẫu cây đã được TS. Nguyễn Quốc Bình, Bảo tàng Thiên nhiên Việt Nam, Viện Hàn lâm Khoa học và Khoa học Công nghệ Việt Nam, giám định tên khoa học. Mẫu tiêu bản số VMN-B0001369 được lưu giữ tại Viện Hóa học các Hợp chất thiên nhiên, Viện Hàn lâm Khoa học và Công nghệ Việt Nam.

\subsection{Hóa chất và thiết bị}

Sắc kí lớp mỏng được thực hiện trên bản mỏng tráng sã̃n DC-Aluofolien $60 \mathrm{~F}_{254}$ (Merck 1,05715). Phát hiện vệt chất bằng đèn tử ngoại ở hai bước sóng $254 \mathrm{~nm}$ và $368 \mathrm{~nm}$ hoặc dùng thuốc thử là dung dịch $\mathrm{H}_{2} \mathrm{SO}_{4} 10 \%$, sấy khô rồi hơ nóng từ từ trên bếp điện đến khi hiện màu. Sắc kí lớp mỏng điều chế được thực hiện trên bản mỏng thủy tinh tráng săn Merck $60 \mathrm{~F}_{254}$, kích cỡ $20 \times 20 \mathrm{~cm}$. Sắc kí cột được tiến hành với chất hấp phụ là silicagel Merck, pha thường, cỡ hạt là $0,040-0,063 \mathrm{~mm}(240-430 \mathrm{mesh})$. Điểm nóng chảy được đo trên máy BOTIUS (Heiztisch Mikroskop) của Đức. Phổ khối ion hóa bụi electron (ESI-MS) được đo trên máy AGILENT 1100 LC-MSD Trap spectrometer. Phổ cộng hưởng từ hạt nhân ${ }^{1} \mathrm{H}-\mathrm{NMR}(500 \mathrm{MHz})$ và ${ }^{13} \mathrm{C}-$ NMR (125 MHz) được đo trên máy Bruker AM500-FT-NMR, sử dụng TMS làm chất nội chuẩn.

\subsection{Chiết tách và phân lập các chất}

Thực hiện quá trình chiết kết hợp siêu âm và gia nhiệt $5 \mathrm{~kg}$ bột khô củ sâm đại hành trong dung môi metanol ở $50^{\circ} \mathrm{C},(3$ lần $\times 2$ giờ mỗi lần $)$. Tổng dịch metanol sau đó được cất loại dung môi dưới áp suất giảm thu được $800 \mathrm{~g}$ cao chiết metanol tổng. Hòa tan cao metanol tổng trong nước và lần lượt chiết với các dung môi có độ phân cực tăng dần là $n$-hexan, etyl axetat và $n$ butanol thu được các dịch chiết tương ứng. Cất loại dung môi dưới áp suất giảm thu được $40 \mathrm{~g}$ cao chiết n-hexan (kí hiệu $\mathrm{EB} / \mathrm{A}), 53 \mathrm{~g}$ cao chiết etyl axetat (kí hiệu $\mathrm{EB} / \mathrm{B}$ ) và $67 \mathrm{~g}$ cao n-butanol (kí hiệu $\mathrm{EB} / \mathrm{C}$ ). Phần dịch nước còn lại được tiến hành sắc kí trên cột dianion với dung môi rửa giải là metanol, sau khi cô quay để thu hồi dung môi thu được $12 \mathrm{~g}$ cặn EB/D.

Cặn EB/B (53 g) được tiến hành phân lập trên sắc kí cột với chất hấp phụ silicagel pha thường, hệ dung môi rửa giải n-hexan:axeton (từ $90: 1$ đến $1: 1, \mathrm{v} / \mathrm{v}$ ) thu được 7 phân đoạn (kí hiệu từ $\mathrm{B} 1$ đến $\mathrm{B} 7)$. Phân đoạn $\mathrm{B} 5$ được tiếp tục sắc kí cột trên silicagel pha đảo với hệ dung môi rửa giải $\mathrm{MeOH}: \mathrm{H}_{2} \mathrm{O}(6: 1, \mathrm{v} / \mathrm{v})$ thu được hợp chất 1 (12 mg, bột màu trắng). Sau khi tinh chế phân đoạn $\mathrm{B} 3$ bằng sắc kí lớp mỏng điều chế với hệ dung môi rửa giải $n$-hexan:xeton $(4: 1, \mathrm{v} / \mathrm{v})$ thu được chất hợp 3 (6 mg, bột màu trắng).

Cặn $\mathrm{EB} / \mathrm{D}(12 \mathrm{~g})$ được sắc kí trên cột dianion với dung môi rửa giải theo nồng độ tăng dần $\mathrm{MeOH}\left(0 \% \mathrm{MeOH}: 100 \% \mathrm{H}_{2} \mathrm{O} ; 25 \% \mathrm{MeOH}, 50 \% \mathrm{MeOH}, 75 \% \mathrm{MeOH}\right.$, và $\left.100 \% \mathrm{MeOH}\right)$ thu được 5 phân đoạn (kí hiệu từ $\mathrm{C} 1$ đến $\mathrm{C} 5$ ). Phân đoạn $\mathrm{C} 3$ tiếp tục được tiến hành sắc kí cột trên silica gel pha thường và rửa giải với hệ dung môi $\mathrm{CHCl}_{3}: \mathrm{MeOH}: \mathrm{H}_{2} \mathrm{O}(5: 1: 0,15, \mathrm{v} / \mathrm{v} / \mathrm{v})$ thu được 3 phân đoạn nhỏ hơn kí hiệu từ $\mathrm{C} 3.1$ đến $\mathrm{C} 3.3$. Từ phân đoạn $\mathrm{C} 3.2$, bằng sắc kí cột trên silica gel pha đảo và rửa giải bằng hệ dung môi $\mathrm{MeOH}: \mathrm{H}_{2} \mathrm{O}(1: 1, \mathrm{v} / \mathrm{v})$ thu được chất hợp 2 ( $9 \mathrm{mg}$, bột màu trắng). 


\section{Hợp chất 1: Eleutherinol}

Chất bột màu trắng, m.p. $308-310^{\circ} \mathrm{C}$, ESI-MS $m / z 257[\mathrm{M}+\mathrm{H}]^{+}$

${ }^{1} \mathrm{H}-\mathrm{NMR}(500 \mathrm{MHz}, \mathrm{MeOH}) \delta(\mathrm{ppm}): 6,18(1 \mathrm{H}, \mathrm{s}, \mathrm{H}-3) ; 7,19(1 \mathrm{H}, \mathrm{s}, \mathrm{H}-6) ; 6,56(1 \mathrm{H}, \mathrm{d}$, $J=2,1 \mathrm{~Hz}, \mathrm{H}-7) ; 6,58(1 \mathrm{H}, \mathrm{d}, J=2,1 \mathrm{~Hz}, \mathrm{H}-9) ; 2,37(3 \mathrm{H}, \mathrm{s}, 2-\mathrm{Me}) ; 2,71\left(3 \mathrm{H}, \mathrm{s}, 5-\mathrm{CH}_{3}\right) ; 10,05(1 \mathrm{H}$, s, $8-\mathrm{OH}) ; 10,15(1 \mathrm{H}, \mathrm{s}, 10-\mathrm{OH})$.

${ }^{13} \mathrm{C}-\mathrm{NMR}(125 \mathrm{MHz}, \mathrm{MeOH}) \delta(\mathrm{ppm}): 156,8$ (C-1); 163,5 (C-2); 112,1 (C-3); 178,6 (C-4); 116,3 (C-4a); 134,4 (C-5); 124,8 (C-6); 138,7 (C-6a); 102,9 (C-7); 156,9 (C-8); 101,2 (C-9); 159,3 (C-10); 107,2 (C-10a); 19,4 (2-Me); 23,0 (5-Me).

\section{Họpp chất 2: Eleutherinoside A}

Chất bột màu trắng, m.p. 275278 ESI-MS m/z 417 [M-H]'

${ }^{1} \mathrm{H}-\mathrm{NMR}\left(500 \mathrm{MHz}, \mathrm{DMSO}-\mathrm{d}_{6}\right), \delta(\mathrm{ppm}): 6,18(1 \mathrm{H}, \mathrm{s}, \mathrm{H}-3) ; 7,27(1 \mathrm{H}, \mathrm{s}, \mathrm{H}-6) ; 6,89(1 \mathrm{H}, \mathrm{d}$, $J=2,1 \mathrm{~Hz}, \mathrm{H}-7) ; 6,66(1 \mathrm{H}, J=2,1 \mathrm{~Hz}, \mathrm{H}-9) ; 2,35(3 \mathrm{H}, \mathrm{s}, 2-\mathrm{Me}) ; 2,70\left(3 \mathrm{H}, \mathrm{s}, 5-\mathrm{CH}_{3}\right) ; 4,98(1 \mathrm{H}, \mathrm{d}$, $J=7,6 \mathrm{~Hz}, \mathrm{H}-1$ '); $3,24(1 \mathrm{H}, \mathrm{t}, J=7,6 \mathrm{~Hz}, \mathrm{H}-2$ '); 3,36 (1H, m, H-3'); $3,19(1 \mathrm{H}, \mathrm{t}, J=8,3 \mathrm{~Hz}, \mathrm{H}-$ 4'); 3,28 (1H, m, H-5'); 3,49 (1H, dd, $\left.J=5,5 \mathrm{~Hz}, 11.4 \mathrm{~Hz}, \mathrm{H}-6{ }^{\prime} \alpha\right) ; 3,69(1 \mathrm{H}, \mathrm{dd}, J=1,2 \mathrm{~Hz}$, $11,4 \mathrm{~Hz}, \mathrm{H}-6$ ' $\beta$ ).

${ }^{13} \mathrm{C}-\mathrm{NMR}\left(125 \mathrm{MHz}, \mathrm{DMSO}-\mathrm{d}_{6}\right), \delta_{\mathrm{C}}, \mathrm{ppm}: 156,3(\mathrm{C}-1) ; 163,7$ (C-2); 112,1 (C-3); 178,5 (C-4); 117,1 (C-4a); 134,7 (C-5); 125,5 (C-6); 138,1 (C-6a); 101,5 (C-7); 158,5 (C-8); 103,1 (C9); 156,5 (C-10); 108,7 (C-10a); 19,5 (C-2-Me); 23,0 (C-5-Me); 100,0 (C-1'); 73,2 (C-2'); 77,1 (C-3'); 69,6 (C-4'); 76,7 (C-5'); 60,6 (C-6').

\section{Họ̣p chất 3: Deoxyerythrolaccin (1,3,6-trihydroxy-8-methyl-anthraquinone)}

Chất bột màu trắng $(12 \mathrm{mg})$, m.p. $325-327^{\circ} \mathrm{C}$, ESI-MS $m / z 271[\mathrm{M}+\mathrm{H}]^{+}$.

${ }^{1} \mathrm{H}-\mathrm{NMR}(500 \mathrm{MHz}, \mathrm{MeOH}) \delta(\mathrm{ppm}): 6,40(1 \mathrm{H}, \mathrm{d}, J=2,7 \mathrm{~Hz}, \mathrm{H}-2) ; 6,94(1 \mathrm{H}, \mathrm{d}, J=2,7 \mathrm{~Hz}$, $\mathrm{H}-4) ; 7,31(1 \mathrm{H}, \mathrm{d}, J=2,7 \mathrm{~Hz}, \mathrm{H}-5) ; 6,80(1 \mathrm{H}, \mathrm{d}, J=2,7 \mathrm{~Hz}, \mathrm{H}-7) ; 2,61\left(3 \mathrm{H}, 8-\mathrm{CH}_{3}\right)$.

${ }^{13} \mathrm{C}-\mathrm{NMR}(125 \mathrm{MHz}, \mathrm{MeOH}) \delta(\mathrm{ppm}): 166,2$ (C-1); 109,3 (C-2); 165,3 (C-3); 108,2 (C-4); 113,3 (C-5); 163,0 (C-6); 125,6 (C-7); 146,6 (C-8); 189,5 (C-9); 184,1 (C-10); 111,6 (C-11); 135,8 (C-12); 138,3 (C-13); 124,3 (C-14); 24,2 (8- $\left.\mathrm{CH}_{3}\right)$.

\section{KẾT QUẢ VÀ THẢO LUẬN}

Bằng phương pháp sắc kí cột kết hợp trên silicagel pha thường, pha đảo và trên dianion với các hệ dung môi rửa giải khác nhau, từ cặn chiết etyl axetat của củ sâm đại hành, chúng tôi đã phân lập được ba hợp chất sạch kí hiệu là 1,2 và 3 .

Hợp chất 1 thu được dưới dạng chất bột màu trắng, có khối lượng phân tử 256 dựa vào pic ion giả phân tử tại $\mathrm{m} / z 257[\mathrm{M}+\mathrm{H}]^{+}$trên phổ ESI-MS, tương ứng với công thức phân tử $\mathrm{C}_{15} \mathrm{H}_{12} \mathrm{O}_{4}$. Phổ ${ }^{1} \mathrm{H}-\mathrm{NMR}$ của 1 có các tín hiệu đặc trưng của 2 proton thơm ghép cặp meta tại $\delta_{\mathrm{H}}$ $6,56(1 \mathrm{H}, \mathrm{d}, J=2,1 \mathrm{~Hz}, \mathrm{H}-7)$ và $\delta_{\mathrm{H}} 6,58(1 \mathrm{H}, \mathrm{d}, J=2,1 \mathrm{~Hz}, \mathrm{H}-9)$; một tín hiệu singlet của 1 proton olefin tại $\delta_{\mathrm{H}} 6,18(1 \mathrm{H}, \mathrm{s}, \mathrm{H}-3)$ và một tín hiệu singlet khác của 1 proton thơm tại $\delta_{\mathrm{H}} 7,19$ $(1 \mathrm{H}, \mathrm{s}, \mathrm{H}-6)$. Ngoài ra trên phổ ${ }^{1} \mathrm{H}-\mathrm{NMR}$ của 1 còn xuất hiện 2 tín hiệu singlet của proton thuộc 2 nhóm methyl tại $\delta_{\mathrm{H}} 2,37\left(3 \mathrm{H}, \mathrm{s}, \mathrm{CH}_{3}-2\right)$ và $\delta_{\mathrm{H}} 2,71\left(3 \mathrm{H}, \mathrm{s}, \mathrm{CH}_{3}-5\right)$. Tương ứng trên phổ ${ }^{13} \mathrm{C}$ NMR và DPET chỉ ra sự có mặt của 15 vạch tín hiệu tương ứng với 15 nguyên tử cacbon, trong 
đó có 2 tín hiệu của cacbon thuộc nhóm methyl tại $\delta_{\mathrm{C}} 19,4\left(\mathrm{CH}_{3}-2\right)$ và $\delta_{\mathrm{C}} 22,97 \quad\left(\mathrm{CH}_{3}-5\right), 4$ tín hiệu của cacbon thuộc nhóm metin tại $\delta_{\mathrm{C}} 112,1$ (C-3), 124,8 (C-6), 102,9 (C-7), 101,1 (C-9), 8 tín hiệu của $C$ bậc 4 và một tín hiệu của cacbon thuộc nhóm cacbonyl vòng lacton tại $\delta_{C}$ 178,6 . Các dữ liệu phổ thu được của 1 phù hợp với dạng khung naphthopyrone khá phổ biến trong các loài Eleutherine [7]. So sánh các dữ liệu phổ trên với các số liệu phổ đã công bố, hợp chất 1 được xác định là eleutherinol và có công thức như trong hình vẽ 1 [8].
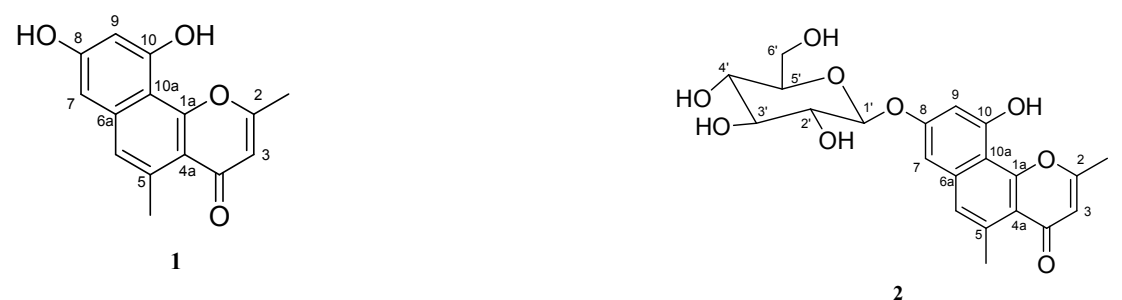<smiles>Cc1cc(O)ccc1C(=O)C1=CC(O)C=C(O)C1=O</smiles>

Hình 1. Cấu trúc của các hợp chất 1-3 phân lập từ củ sâm đại hành.

Phổ ${ }^{1} \mathrm{H}-\mathrm{NMR}$ của hợp chất 2 cho thấy hợp chất này cũng chứa khung naphthopyrone được đặc trưng bởi hai proton thơm ghép cặp meta với nhau tại $\delta_{\mathrm{H}} 6,89(1 \mathrm{H}, \mathrm{d}, \mathrm{H}-7)$ và $\delta_{\mathrm{H}} 6,66(1 \mathrm{H}$, $\mathrm{d}, \mathrm{H}-9)$ với hằng số tương tác $J$ đều bằng $2,1 \mathrm{~Hz}$; một tín hiệu singlet tại $\delta_{\mathrm{H}} 6,18(1 \mathrm{H}, \mathrm{s}, \mathrm{H}-3)$ và một tín hiệu singlet của 1 proton thơm khác ở $\delta_{\mathrm{H}} 7,27(1 \mathrm{H}, \mathrm{s}, \mathrm{H}-6)$; ngoài ra còn có 2 nhóm $\mathrm{CH}_{3}$ tại $\delta_{\mathrm{H}} 2,35(3 \mathrm{H}, \mathrm{s}, \mathrm{Me}-2)$ và $\delta_{\mathrm{H}} 2,70(3 \mathrm{H}, \mathrm{s}, \mathrm{Me}-5)$. Nhưng khác với 1 , trên phổ ${ }^{1} \mathrm{H}-\mathrm{NMR}$ của hợp chất 2 còn thấy sự xuất hiện của một cấu tử đường glucose thông qua sự xuất hiện của các tín hiệu proton của các nhóm metin có độ chuyển dịch hóa học nằm trong khoảng từ 3,19 đến 3,69 ppm và 1 tín hiệu proton anome tại $\delta_{\mathrm{H}} 4,98(\mathrm{~d}, J=7,6 \mathrm{~Hz})$. Phổ ${ }^{13} \mathrm{C}-\mathrm{NMR}$ và $\mathrm{DEPT}$ của 2 cũng cho thấy, ngoài sự xuất hiện 15 tín hiệu của khung naphthopyrone tương tự như của hợp chất 1 , hợp chất 2 còn thấy xuất hiện thêm các tín hiệu của 5 nguyên tử cacbon có độ chuyển dịch hóa học nằm trong vùng từ 60,6 đến $77,1 \mathrm{ppm}$ và 1 tín hiệu của cacbon anome tại $\delta_{\mathrm{C}} 100$ ppm, điều này cho thấy trong cấu trúc của 2 còn có sự xuất hiện của một cấu tử đường glucose gắn vào khung naphthopyrone. Phổ khối lượng phun mù điện tử của 2 cho thấy có pic ion giả phân tử tại $\mathrm{m} / \mathrm{z}=417[\mathrm{M}-\mathrm{H}]^{-}$chứng tỏ 2 có khối lượng phân tử 418 , nhiều hơn 1 một cấu tử đường glucose. Các liên kết giữa $\mathrm{C}$ và $\mathrm{H}$ được chỉ ra ở phổ $\mathrm{HMQC}$ đều phù hợp với các kết luận trên. Cấu hình dạng $\beta$ của $\mathrm{C}$ anomeric được xác định dựa vào hằng số tương tác $\mathrm{J}_{\mathrm{H}-\mathrm{1}^{\prime}, \mathrm{H}-2},=7,6$ Hz. Phổ HMBC của 2 cho thấy sự tương tác giữa $\mathrm{H}-1$ ' $\left(\delta_{\mathrm{H}} 4,98\right)$ với $\mathrm{C}-8\left(\delta_{\mathrm{C}} 158,5\right)$, diều này khẳng định cấu tử đường glucose được gắn vào vị trí $\mathrm{C}-8$ của khung naphthopyrone. Từ các dữ liệu trên, kết hợp với tài liệu tham khảo [9], hợp chất $\mathbf{2}$ được xác định là eleutherinoside $\mathrm{A}$ và có cấu trúc như ở hình 1 . Hợp chất eleutherinoside $\mathrm{A}$ thể hiện có hoạt tính chống đái đường tốt với giá trị $\mathrm{IC}_{50}$ là $0,5 \mathrm{mM}[10]$.

Hợp chất 3 thu được dưới dạng chất bột màu trắng, phổ khối cho pic ion giả phân tử tại $m / z 271[\mathrm{M}+\mathrm{H}]^{+}$tương ứng với công thức phân tử $\mathrm{C}_{15} \mathrm{H}_{10} \mathrm{O}_{5}$. Phổ ${ }^{1} \mathrm{H}-\mathrm{NMR}$ của 3 có tín hiệu của 2 cặp proton thơm ở vị trí meta với nhau tại $\delta_{\mathrm{H}} 6,40(1 \mathrm{H}, \mathrm{d}, J=2,7 \mathrm{~Hz}, \mathrm{H}-2), 6,94(1 \mathrm{H}, \mathrm{d}, J=2,7$ 
$\mathrm{Hz}, \mathrm{H}-4), 7,31(1 \mathrm{H}, \mathrm{d}, J=2,7 \mathrm{~Hz}, \mathrm{H}-5)$ và $6,80(1 \mathrm{H}, \mathrm{d}, J=2,7 \mathrm{~Hz}, \mathrm{H}-7)$; một tín hiệu singlet tại $\delta_{\mathrm{H}} 2,61 \mathrm{ppm}$ tương ứng với sự xuất hiện của một nhóm $\mathrm{CH}_{3}$. Trên phổ ${ }^{13} \mathrm{C}-\mathrm{NMR}$ và $\mathrm{DEPT}$ cho thấy tín hiệu của 15 nguyên tử $\mathrm{C}$, trong đó có 12 nguyên tử $\mathrm{C}$ vòng thơm dao động trong khoảng 108,2 ppm cho đến 166,2 ppm và 2 nguyên tử cacbon của 2 nhóm cacbonyl tại $\delta_{\mathrm{C}} 184,9(\mathrm{C}-10)$ và $\delta_{\mathrm{C}} 189,5$ (C-9) gợi ý cho thấy sự có mặt của bộ khung anthraquinone khá phổ biến ở các loài Eleutherine; ngoài ra trên phổ DEPT còn cho thấy tín hiệu của 1 nhóm $\mathrm{CH}_{3}$ gắn với vòng thơm ở $\delta_{\mathrm{C}} 24,2 \mathrm{ppm}(8-\mathrm{Me})$. Các dữ kiện phổ này đều phù hợp với tín hiệu cho trên phổ 2 chiều $\mathrm{HMQC}$ và $\mathrm{HMBC}$. Trên phổ $\mathrm{HMQC}$ thấy tương tác của $\mathrm{C}-\mathrm{H}$ liên kết trực tiếp với nhau gồm các tương tác $\mathrm{C}-2$ với $\mathrm{H}-2, \mathrm{C}-4$ với $\mathrm{H}-4, \mathrm{C}-5$ với $\mathrm{H}-5, \mathrm{C}-7$ với $\mathrm{H}-7$. Phổ $\mathrm{HMBC}$ cho tín hiệu của tương tác giữa $\mathrm{C}$ và $\mathrm{H}$ cách nhau 3 liên kết gồm các tương tác của $\mathrm{H}-2$ với $\mathrm{C}-1, \mathrm{C}-3, \mathrm{C}-4, \mathrm{C}-11$; $\mathrm{H}-4$ với $\mathrm{C}-2, \mathrm{C}-3, \mathrm{C}-10, \mathrm{C}-11 ; \mathrm{H}-5$ với $\mathrm{C}-6, \mathrm{C}-7, \mathrm{C}-10, \mathrm{C}-14 ; \mathrm{H}-7$ với $\mathrm{C}-5, \mathrm{C}-6, \mathrm{C}-14$ và $8-\mathrm{CH}_{3}$; đặc biệt, tương tác của proton của nhóm $\mathrm{CH}_{3}-8$ với các cacbon $\mathrm{C}-7, \mathrm{C}-8, \mathrm{C}-14$ đã cho thấy nhóm $\mathrm{CH}_{3}$ được gắn vào vị trí $\mathrm{C} 8$ của bộ khung anthraquinone. So sánh các dữ liệu thu được với các số liệu đã công bố thấy hoàn toàn trùng khớp [11]. Do vậy, hợp chất 3 được xác định là 1,3,6trihydroxy-8-methyl-anthraquinone, có công thức như hình 1.

\section{KẾT LUẬN}

Từ cặn chiết etyl axetat và cặn chiết nước của củ Sâm đại hành (Eleutherine bulbosa) đã phân lập được hai dẫn xuất của naphthopyrone và một dẫn xuất của anthraquinone. Bằng các phương pháp phổ kết hợp gồm ESI-MS và $1 \mathrm{D}-\mathrm{NMR}, 2 \mathrm{D}-\mathrm{NMR}$, cấu trúc của 3 hợp chất này đã được xác định là eleutherinol, eleutherinoside $\mathrm{A}$ và 1,3,6-trihydroxy-8-methyl-anthraquinone.

Lời cảm ơn. Công trình này được hoàn thành với sự tài trợ kinh phí của Đề tài cấp Viện Hàn lâm Khoa học và Công nghệ Việt Nam, mã số đề tài VAST 04.01/12-13. Tác giả xin cảm ơn TS. Nguyễn Quốc Bình đã thu hái và giám định mẫu thực vật.

\section{TÀI LIỆU THAM KHẢO}

1. Võ Văn Chi - Từ điển cây thuốc Việt Nam, Nhà xuất bản Y học, 1997, tr. 1029.

2. Đỗ Tất Lợi - Những cây thuốc và vị thuốc Việt Nam, Nhà xuất bản Y học, 2004, tr. 145.

3. Alves Tania Maria Almeida, Kloos Helmut et al. - Eleutherinone, a novel fungitoxic naphthoquinone from Eleutherine bulbosa (Iridaceae), Memorias do Instituto Oswaldo Cruz 98 (5) (2003) 709-712.

4. Gallo Francesca R., Palazzino Giovanna et al. - Polyketides from Eleutherine bulbosa, Natural Product Research 24 (16) (2010) 1578-1586.

5. Bianchi C, Ceriotti G. - Chemical and pharmacological investigations of constituents of Eleutherine bulbosa (Miller) Urb. (Iridaceae), Journal of Pharmaceutical Sciences 64 (8) (2010) 1305-1308.

6. Nguyễn Thị Hồng Vân, Đỗ Thị Thanh Huyền, Ngọ Thị Phương, Nguyễn Thị Hoàng Anh, Bùi Kim Anh, Nguyễn Mạnh Cường, Nguyễn Tuấn Anh, Lê Minh Hà - Một số dẫn xuất naphthopyran phân lập từ củ sâm đại hành (Eleutherine bulbosa) ở Việt Nam, Tạp chí Khoa học và Công nghệ 50 (3A) (2012) 8-14.

7. Ah-Reum HAN, aHye-Young MIN, Joo-Won NAM, Na-Youn LEE, aAdam WIRYAWAN, Wahyu SUPRAPTO, Sang Kook LEE, Kang Ro LEE, and Eun-Kyoung SEO - Identification of a New Naphthalene and Its Derivatives from the Bulb of 
Eleutherine americanawith Inhibitory Activity on Lipopolysaccharide-Induced Nitric Oxide Production, Chem. Pharm. Bull. 56 (9) (2008) 1314-1316.

8. Byung-Sun MIN, Jong-Pill LEE, Min-Kyun NA, Ren-Bo AN, Sang-Myung LEE, Hyeong-Kyu LEE, KiHwan BAE, and Sam-Sik KANG - A New Naphthopyrone from the Root of Pleuropterus ciliinervis, Chem. Pharm. Bull. 51 (11) (2003) 1322-1324.

9. Tomohiro Ieyama, Maria D.P.T. Gunawan-Puteri, Jun Kawabata - $\alpha$-Glucosidase inhibitors from the bulb ofEleutherine americana, Food Chemistry 128 (2011) 308-311.

10. Sompol Paramapojn, Markus Ganzera, Wandee Gritsanapan, Hermann Stuppner Analysis of naphthoquinone derivatives in the Asian medicinal plant Eleutherine americanaby RP-HPLC and LC-MS, Journal of Pharmaceutical and Biomedical Analysis 47 (2008) 990-993.

11. Dieudonne Ngamga, Maurice D. Awouafack, Pierre Tane, Merhatibeb Bezabih, Berhanu M. Abegaz - Two new anthraquinones fromGladiolus psittascinus, Biochemical Systematics and Ecology 35 (2007) 709-713.

\author{
ABSTRACT \\ ISOLATION AND STRUCTURAL ELUCIDATION OF SOME NAPHTHOPYRONE \\ AND ANTHRAQUINONE DERIVATIVES FROM Eleutherine bulbosa \\ Nguyen Thi Hong Van ${ }^{1,}$, Luu Tuan Anh ${ }^{1}$, Le Minh Ha ${ }^{1}$, Ngo Thi Phuong ${ }^{1}$, \\ Trinh Anh Vien ${ }^{2}$, Pham Quoc Long ${ }^{1}$ \\ ${ }^{1}$ Institute of Natural Products Chemistry, VAST, 18 Hoang Quoc Viet, Cau Giay, Hanoi \\ ${ }^{2}$ Industrial University of Hochiminh city, Thanh Hoa city \\ *Email:van762004@yahoo.com
}

Two naphthopyrone derivatives, including eleutherinol (1) and eleutherinoside A (2) have been isolated from the ethyl acetate extract of Eleutherine bulbosa together with an anthraquinone derivative 1,3,6-trihydroxy-8-methyl-anthraquinone (3). The structures of these compounds were determined by spectroscopic data analysis.

Keywords: naphthopyrone, eleutherinol, eleutherinoside A, anthraquinone, 1,3,6-trihydroxy-8methyl-anthraquinone, Eleutherine bulbosa. 